\title{
Hounal of Biomedicine
}

2017; 2: 120-123. doi: 10.7150/jbm.20986

Review

\section{The Endothelial Glycocalyx: Visualization and Measurement}

\author{
Hongyan Kang ${ }^{\bowtie}$ \& Xiaoyan Deng
}

Key Laboratory for Biomechanics and Mechanobiology of Ministry of Education, School of Biological Science and Medical Engineering, Beihang University, Beijing 100191, China.

\author{
$\triangle$ Corresponding author: Hongyan Kang, hongyankang@buaa.edu.cn \\ (C) Ivyspring International Publisher. This is an open access article distributed under the terms of the Creative Commons Attribution (CC BY-NC) license \\ (https://creativecommons.org/licenses/by-nc/4.0/). See http://ivyspring.com/terms for full terms and conditions.
}

Received: 2017.05.12; Accepted: 2017.06.20; Published: 2017.08.20

\begin{abstract}
The endothelial glycocalyx is a hair-like surface layer mainly consisted of glycosaminoglycans (GAGs) that includes heparan sulfate (HS), chondroitin sulfate (CS), and hyaluronic acid (HA) and its bearing core proteins (syndecan, glypican, and perlecan). This mini review presents the visualization and measurement techniques of the endothelial glycocalyx up to now, providing insight to the diagnosis, treatment, and protection of the cardiovascular disease.
\end{abstract}

Key words: Glycocalyx, transmission electron microscopy, intravital microscopy, confocal laser scanning microscopy, two-photon laser scanning microscopy.

\section{Introduction}

The endothelial glycocalyx is a hair-like surface layer mainly consisted of glycosaminoglycans (GAGs) that includes heparan sulfate (HS), chondroitin sulfate (CS), and hyaluronic acid (HA) and its bearing core proteins (syndecan, glypican, and perlecan) $[1,2]$. Due to its special location, the endothelial glycocalyx plays important roles in mechanotransduction, the regulation of vascular permeability, the rheological behavior of the microcirculation, and the adhesion of blood cells to the endothelium. The perturbation of the endothelial glycocalyx will be directly responsible for several pathophysiological sequelae such as diabetes [3], ischemia/reperfusion injury [4], atherosclerosis [5], and sepsis [6]. Usually, the structure of glycocalyx was observed after enzymatic treatment to underlie the functional modifications [7]. The present review focused on the reliable visualization and measurement techniques that could be used to determine the structure of the endothelial glycocalyx, as outlined in table 1, which covers the transmission electron microscopy (TEM), intravital microscopy (IM), microparticle image velocimetry
$(\mu$-PIV), orthogonal polarization spectral imaging (OPS), side-stream dark field imaging (SDF), confocal laser scanning microscopy (CLSM), and two-photon laser scanning microscopy (TPLSM).

Table 1 The visualization and measurement techniques of the endothelial glycocalyx (GCX)

\begin{tabular}{|c|c|c|c|}
\hline $\begin{array}{l}\text { GCX visualization and measurement } \\
\text { techniques }\end{array}$ & $\begin{array}{l}\text { In vivo/in } \\
\text { vitro/ex vivo }\end{array}$ & $\begin{array}{l}\text { Direct/ } \\
\text { indirect }\end{array}$ & References \\
\hline $\begin{array}{l}\text { Transmission electron microscopy } \\
\text { (TEM) }\end{array}$ & In vitro & Direct & {$[8-14]$} \\
\hline Intravital microscopy (IM) & In vivo & Indirect & [15-18] \\
\hline $\begin{array}{l}\text { Microparticle image velocimetry } \\
(\mu \text {-PIV) }\end{array}$ & ex vivo & Indirect & {$[19,20]$} \\
\hline $\begin{array}{l}\text { Orthogonal polarization spectral } \\
\text { imaging (OPS) }\end{array}$ & In vivo & Indirect & [21] \\
\hline Side-stream dark field imaging (SDF) & In vivo & Indirect & [22] \\
\hline $\begin{array}{l}\text { Confocal laser scanning microscopy } \\
\text { (CLSM) }\end{array}$ & In vitro & Direct & [23-25] \\
\hline $\begin{array}{l}\text { Two-photon laser scanning } \\
\text { microscopy (TPLSM) }\end{array}$ & ex vivo & Direct & {$[26,27]$} \\
\hline
\end{tabular}




\section{Transmission electron microscopy (TEM)}

Since the first image of the endothelial glycocalyx was obtained by TEM [8], this technique has been developed in the dye selection, the sample fixation methods, and the dehydration. Ruthenium red staining in combination with glutaraldehyde/ osmium tetroxide fixation has been widely used in the early stage [9]. However, the staining has its own limitation [10]. For example, ruthenium red may not have access to the whole glycocalyx layer due to its relatively large molecular size. Moreover, ruthenium red may change the geometry of the glycocalyx by electrostatic effects. In order to overcome these limitations, Haldenby and coworkers [11] tried alcian blue, a smaller molecule, in measuring the thickness of the endothelial glycocalyx on the surface of the rabbit thoracic aorta. The results showed a thickness of about $60 \mathrm{~nm}$, which was better than the results of 20 $\mathrm{nm}$ obtain by ruthenium red staining. In addition to the dye selection, the sample fixation should be another factor affecting the dimensional quantification of endothelial glycocalyx. Chappell et al. [12] quantified the thickness of the human umbilical vein endothelial cell glycocalyx under both perfusion-fixed and immersion-fixed conditions. They found that perfusion fixation by supplement with plasma protein was better in maintaining the structure stability of the dynamic glycocalyx. On the contrary, the glycocalyx was absent by immersion fixation. More recently, Ebong et al. [13] imaged the bovine aortic endothelial glycocalyx in vitro by rapid freezing/freeze substitution transmission electron microscopy, taking advantage of the high spatial resolution of the TEM and the capability to preserve the hydrated configuration of the glycocalyx (GCX). The thickness obtained was approximate $11 \mu \mathrm{m}$, which was better than the $0.04 \mu \mathrm{m}$ from the conventional TEM. It should be noted that even the most recently developed preparation and staining techniques may have side effects on the delicate surface structures of the endothelial glycocalyx, and TEM cannot obtain the dimensional results in vivo [14].

\section{Intravital microscopy (IM)}

The conventional IM was based on the binding of fluorescently labelled lectins to polysaccharide side chains of the glycoproteins and proteoglycans [15]. Measurement of fluorescence intensity allows a semi-quantification of the endothelial glycocalyx. However, due to optical limitations of IM, the fluorescence could not outline the entire glycocalyx to allow a precise measurement of its geometry [16]. Vink and Duling [17] took advantage of an alternative approach to label the flowing plasma by FITC-dextran and identify plasma exclusion. The results demonstrated the existence of a continuous glycocalyx with a thickness of about $0.4-0.5 \mu \mathrm{m}$ in capillaries of the hamster cremaster by subtracting the width of the labelled plasma column from the anatomical diameter. More recently, Kataoka et al. [18] investigated the binding ability of different FITC labelled lectins including Dolichos biflorus agglutinin, Canavalia ensiformis agglutinin, Arachis hypogaea (Peanut) agglutinin, Ricinus communis agglutinin, Ulex europaeus agglutinin, Glycine max (soybean) agglutinin, Triticum vulgaris (wheat germ) agglutinin to the endothelial glycocalyx and found that FITC-wheat germ agglutinin (FITC-WGA) was the best to label the glycocalyx in vivo compared with other lectins. In all, IM was mostly used in microvessels (no larger than $15 \mu \mathrm{m}$ ) that are thin and transparent to observe the flowing blood cells, and the dimensional prediction of the endothelial glycocalyx was indirect.

\section{Microparticle image velocimetry ( $\mu$-PIV)}

In order to enhance the spatial resolution of IM, Damiano and colleagues [19] tried to measuring the velocity profiles in the red cell-depleted plasma layer near the endothelial lining in mouse cremaster muscle venules by using $\mu$-PIV. The key point of this technology should be the fluorescent particle selection, and a linear regression analysis was performed on the $\mu$-PIV data. The distance from the vessel wall where the linear regression extrapolated to zero velocity was taken as the glycocalyx thickness. Based on a detailed three dimensional analysis of the local fluid dynamics, they estimated a thickness of about $500 \mathrm{~nm}$ for an impermeable endothelial surface layer. By using the same $\mu$-PIV technique, Potter et al. [20] showed a hydrodynamically relevant endothelial glycocalyx observed in microvessels in vivo (0.52 \pm $0.28 \mu \mathrm{m}$ thickness) was absent from human umbilical vein $(0.03 \pm 0.04 \mu \mathrm{m}$ thickness $)$ and bovine aortic (0.02 $\pm 0.04 \mu \mathrm{m}$ thickness) endothelial cells maintained under standard cell culture conditions in vitro. In light of these findings, previous results and conclusions drawn from cultured endothelial cells in vitro in the areas of mechnotransduction, permeability, inflammation, and pathology should be carefully reconsidered.

\section{Orthogonal polarization spectral imaging (OPS) and side-stream dark field imaging (SDF)}

The novel imaging techniques developed for microcirculation glycocalyx visualization are OPS and 
SDF. The former is based on a linear theory model that could be described as follows. In brief, endothelial glycocalyx limits the movement of red blood cells to the vessel wall. In contrast, white blood cells which are more rigid compress the endothelial glycocalyx as they pass through the lumen. Hence, there is a transient "widening" of the red blood cells following the white blood cell passage. The glycocalyx thickness was determined as the width change of the red blood cells divided by 2 . This OPS technique has been used successfully in sublingual microvascular glycocalyx measurement in 24 healthy men volunteers [21]. In order to obtain clear images of the endothelial glycocalyx, SDF was developed to detect much deeper microvessels [22]. Nevertheless, both the OPS and SDF could be only used to measure the endothelial glycocalyx limited to the microcirculation. Moreover, the thickness prediction was indirect as well.

\section{Confocal laser scanning microscopy (CLSM)}

For the artery endothelial glycocalyx visualization and measurement, CLSM and TPLSM have the advantages in optical sectioning, high resolution, and three-dimensional reconstruction. The endothelial glycocalyx could be labeled by several approaches, mostly using fluorescent probe attached lectins which could bind the glycosaminoglycan chains. Other labels include antibodies specific for core protein backbones such as syndecan and HS, CS or HA then combined with a respective fluorescently labeled secondary antibody [23]. It should be noted that CLSM is not suitable for imaging of the arterial glycocalyx directly since the resolution decreased significantly at higher depths $(>40 \mu \mathrm{m})$ due to increased scattering of signal when light penetrated into the arterial wall [24]. In order to overcome this limitation, Kang et al. [25] tried cryosection of the blood vessel to 5-6 $\mu \mathrm{m}$ slices then labeled the cross-sections with WGA-FITC, realizing the visualization and quantification of the endothelial glycocalyx by CLSM.

\section{Two-photon laser scanning microscopy (TPLSM)}

Another promising technique suitable for visualize the arterial glycocalyx directly is TPLSM which used long wavelength red photons to excite the fluorophore instead of the blue photon as used in conventional fluorescence excitation [26]. In this manner, the scattering could be reduced, and hence, the penetration depth could be increased. Moreover, since the consequent fluorescence only occurs at the focal point of the illumination core, the bleaching and phototoxicity is just limited to this focal position and the resolution is kept through the whole depth. By using TPLSM, Megens et al. [27] imaged the mouse carotid arterial glycocalyx ex vivo and measured the thickness of endothelial glycocalyx that was about $4.5 \pm 1.0 \mu \mathrm{m}$.

In summary, both TEM and CLSM need sample fixation to keep its original structure, which may compress or distort the configuration of the glycocalyx to some extent. Barker et al. used lectin to label the live human umbilical vein endothelial cells cultured in vitro without fixation and observed the glycocalyx in CLSM successfully, which suggests developing live cell or tissue glycocalyx probes may be a promising direction in future study. IM, $\mu$-PIV, OPS and SDF predict the thickness of the endothelial glycocalyx indirectly and are limited to the microcirculation. In contrast, TPLSM with its enhanced penetration depth, good resolution, and low phototoxicity, may be a promising tool in directly visualizing the glycocalyx in larger arteries, both in vivo and ex vivo.

\section{Acknowledgements}

This work is supported by Grants-in-Aid from the National Natural Science Foundation of China (No. 31500763, 11572028).

\section{Competing Interests}

The authors have declared that no competing interest exists.

\section{References}

1. Tarbell JM, Pahakis MY. Mechanotransduction and the glycocalyx. J Intern Med. 2006; 259: 339-50.

2. Zeng Y. Endothelial glycocalyx as a critical signalling platform integrating the extracellular haemodynamic forces and chemical signalling. J Cell Mol Med. 2017.

3. Nieuwdorp M, van Haeften TW, Gouverneur MC, Mooij HL, van Lieshout $\mathrm{MH}$, Levi $\mathrm{M}$, et al. Loss of endothelial glycocalyx during acute hyperglycemia coincides with endothelial dysfunction and coagulation activation in vivo. Diabetes. 2006; 55: 480-6

4. Mulivor AW, Lipowsky HH. Inflammation- and ischemia-induced shedding of venular glycocalyx. Am J Physiol Heart Circ Physiol. 2004; 286: H1672-80.

5. Meuwese MC, Mooij HL, Nieuwdorp M, van Lith B, Marck R, Vink H, et al. Partial recovery of the endothelial glycocalyx upon rosuvastatin therapy in patients with heterozygous familial hypercholesterolemia. J Lipid Res. 2009; 50: 148-53.

6. Steppan J, Hofer S, Funke B, Brenner T, Henrich M, Martin E, et al. Sepsis and major abdominal surgery lead to flaking of the endothelial glycocalix. J Surg Res. 2011; 165: 136-41

7. Zeng Y, Ebong EE, Fu BM, Tarbell JM. The structural stability of the endothelial glycocalyx after enzymatic removal of glycosaminoglycans. Plos One. 2012; 7: e43168.

8. Luft JH. Fine structures of capillary and endocapillary layer as revealed by ruthenium red. Fed Proc. 1966; 25: 1773-83.

9. Janczyk P, Hansen S, Bahramsoltani M, Plendl J. The glycocalyx of human, bovine and murine microvascular endothelial cells cultured in vitro. J Electron Microsc (Tokyo). 2010; 59: 291-8.

10. Pries AR, Secomb TW, Gaehtgens P. The endothelial surface layer. Pflugers Arch. 2000; 440: 653-66. 
11. Haldenby KA, Chappell DC, Winlove CP, Parker KH, Firth JA. Focal and regional variations in the composition of the glycocalyx of large vessel endothelium. J Vasc Res. 1994; 31: 2-9.

12. Chappell D, Jacob M, Paul O, Rehm M, Welsch U, Stoeckelhuber M, et al. The glycocalyx of the human umbilical vein endothelial cell: an impressive structure ex vivo but not in culture. Circ Res. 2009; 104: 1313-7.

13. Ebong EE, Macaluso FP, Spray DC, Tarbell JM. Imaging the endothelial glycocalyx in vitro by rapid freezing/freeze substitution transmission electron microscopy. Arterioscler Thromb Vasc Biol. 2011; 31: 1908-15.

14. Savery MD, Jiang JX, Park PW, Damiano ER. The endothelial glycocalyx in syndecan-1 deficient mice. Microvasc Res. 2013; 87: 83-91.

15. Witte $\mathrm{S}$. The influence of the fibrinolytic system on the affinity of fibrinogen for the endothelial-plasma interface. Thromb Res. 1988; 52: 111-7.

16. Gretz JE, Duling BR. Measurement uncertainties associated with the use of bright-field and fluorescence microscopy in the microcirculation. Microvasc Res. 1995; 49: 134-40.

17. Vink H, Duling BR. Identification of distinct luminal domains for macromolecules, erythrocytes, and leukocytes within mammalian capillaries. Circ Res. 1996; 79: 581-9.

18. Kataoka H, Ushiyama A, Kawakami H, Akimoto Y, Matsubara S, Iijima T. Fluorescent imaging of endothelial glycocalyx layer with wheat germ agglutinin using intravital microscopy. Microsc Res Tech. 2016; 79: 31-7.

19. Smith ML, Long DS, Damiano ER, Ley K. Near-wall micro-PIV reveals a hydrodynamically relevant endothelial surface layer in venules in vivo. Biophys J. 2003; 85: 637-45.
20. Potter DR, Damiano ER. The hydrodynamically relevant endothelial cell glycocalyx observed in vivo is absent in vitro. Circ Res. 2008; 102: 770-6.

21. Nieuwdorp M, Meuwese MC, Mooij HL, Ince $C$, Broekhuizen LN, Kastelein JJ, et al. Measuring endothelial glycocalyx dimensions in humans: a potential novel tool to monitor vascular vulnerability. J Appl Physiol (1985). 2008; 104: 845-52.

22. den Uil CA, Klijn E, Lagrand WK, Brugts JJ, Ince C, Spronk PE, et al. The microcirculation in health and critical disease. Prog Cardiovasc Dis. 2008; 51: 161-70.

23. Kolarova H, Ambruzova B, Svihalkova Sindlerova L, Klinke A, Kubala L. Modulation of endothelial glycocalyx structure under inflammatory conditions. Mediators Inflamm. 2014; 2014: 694312.

24. Reitsma S, Slaaf DW, Vink H, van Zandvoort MA, oude Egbrink MG. The endothelial glycocalyx: composition, functions, and visualization. Pflugers Arch. 2007; 454: 345-59.

25. Kang H, Sun L, Huang Y, Wang Z, Zhao P, Fan Y, et al. Regional specific adaptation of the endothelial glycocalyx dimension in tail-suspended rats. Pflugers Arch. 2015; 467: 1291-301.

26. van Zandvoort M, Engels W, Douma K, Beckers L, Oude Egbrink M, Daemen $\mathrm{M}$, et al. Two-photon microscopy for imaging of the (atherosclerotic) vascular wall: a proof of concept study. J Vasc Res. 2004; 41: 54-63.

27. Megens RT, Reitsma S, Schiffers PH, Hilgers RH, De Mey JG, Slaaf DW, et al. Two-photon microscopy of vital murine elastic and muscular arteries. Combined structural and functional imaging with subcellular resolution. J Vasc Res. 2007; 44: 87-98. 